\title{
Cómo introducir vectores éticos eficaces en el sistema tecnológico
}

\section{Ramón Queraltó}

Arbor CLXII, 638 (Febrero 1999), 221-240 pp.

Un problema clave de la actual Sociedad tecnológica es el del cambio en los valores éticos. Normalmente, su tratamiento consiste en oponer al sistema tecnológico, desde su exterior, una serie de valores éticos que se consideran deseables e imprescindibles y a los cuales aquél ha de adaptarse. Esta estrategia ha mostrado continuamente su inoperancia real, a causa de la autonomía y de la fuerza propia del sistema tecnológico. Por ello, se propone una nueva Metodología ética, que evite dicha inoperancia; para lo cual se describen, en primer lugar, los rasgos más relevantes de la Sociedad tecnológica y el tipo de racionalidad interna que posee (la racionalidad tecnológica), basada primariamente en la eficacia operativa, para, en segundo lugar, estudiar como criterio de presentación social de los valores éticos dicha eficacia práctica, respecto de los fines del sistema tecnológico. Se trata asi de emplear el principio técnico de eficacia y operatividad como principio de dimensión ética, y hallar semejanzas estructurales entre ambos que justifiquen que el sistema sociotécnico pueda adoptar vectores éticos basados en esta estrategia diferente. Finalmente, se deducen, siguiendo esta Metodología, cuáles podrían ser los valores éticos primordiales que actuaran como vectores éticos eficaces en la Sociedad tecnológica.

\section{Etica, Sociedad y sistema tecnológico: la lógica interna de la situación actual}

La Etica se ha convertido en la actualidad en una de las instancias filosóficas y sociales con más brío dialéctico. En todos los foros de 


\section{Ramón Queraltó}

importancia - académicos, políticos, socioculturales, etc.- se habla de Etica desde los más diversos puntos de vista. Incluso en los últimos años se ha fundado una presuntamente "nueva" rama de la Etica, esto es, la llamada Bioética. Sin embargo, paradójicamente, nunca se ha criticado más la falta de Etica en la vida social y política que en nuestro mundo presente. La dispersión ética contemporánea, o, para otros, el pluralismo ético más o menos radical, se afirma a menudo que es un reflejo de la crisis efectiva de la antigua fortaleza reflexiva y práctica de la dimensión ética en la cultura. Las causas de todo ello son obviamente complejas, teoréticas y pragmáticas, y no es éste el lugar para adentrarse en ellas de modo global. Sí es procedente, no obstante, señalar uno de los factores que condicionan esta situación y que constituye un elemento fundamental determinante, entre otros, de la misma. Se trata del hecho de que hemos entrado ya en la Era de una Sociedad tecnológica.

Al final de la Modernidad, la Historia humana está recogiendo los frutos maduros de la racionalidad científica y ha elevado a límites insospechados uno de los fines constituyentes de dicho período histórico, es decir, la manipulación y transformación de la realidad presuntamente en beneficio del hombre. Esta maximización de los fines pragmáticos del conocimiento científico es obra fundamental de la Tecnología y de su desarrollo imparable en los últimos decenios. La nueva revolución, o sea, la revolución tecnológica, y, en especial, la revolución de las Tecnologías de la información, es el factor estructurante del mundo moderno que posee un alcance social y cultural que no está en discusión ${ }^{1}$. Así, por Sociedad tecnológica hay que entender, en primera aproximación, una organización social en la que el desarrollo y avance tecnológicos desempeña el papel de una causa estructural decisiva de la realidad. En otros términos, la Tecnología ha adquirido el rango de agente histórico-social fundamental de la vida del hombre.

La novedad, al menos relativa, de esta situación incide especialmente en la dimensión ética, pues presenta un conjunto de situaciones y de relaciones sociales que exigen respuestas no convencionales procedentes de instancias éticas ya conocidas y heredadas. Es un lugar común indicar, por ejemplo, que el avance tecnológico ha creado situaciones morales nuevas, que están siempre por delante de los elementos éticos al uso y de los cuerpos legislativos democráticamente establecidos. En definitiva, la Tecnología está planteando una problemática moral ante la cual el hombre de final del milenio se siente perplejo y profundamente dubitativo. 
Ahora bien, la raíz de este asunto no se encuentra en el simple hecho de que una Sociedad tecnológica adopte el desarrollo técnico como principio de estructuración social. Eso sería, a lo sumo, un factor externo, es decir, un elemento que impacta instrumentalmente en el cuerpo social, y es, por ello, relativamente exterior al mismo. Porque, por Sociedad tecnológica, hay que entender también una Sociedad en la que la racionalidad implícita y los valores de todo tipo - positivos y negativos, si se quiere- inmanentes en el fenómeno tecnológico, informan y vertebran la estructura y el funcionamiento de esa Sociedad. Y esto es algo mucho más profundo que un simple "impacto" de la Tecnología en la realidad, según el modo habitualmente utilizado para entender el alcance de tal impacto. Pues se trata, nada menos, del hecho de que el tipo de racionalidad imperante en la vida social y en la organización colectiva se inspira, conscientemente o no, en la forma de racionalidad que es propia de la Tecnología. Y, como consecuencia, que los valores con vigencia social efectiva también se hallen condicionados por tal manera de racionalidad.

Por eso, la demanda contemporánea por la Etica, incluso por más Etica, se ve entrecruzada por la racionalidad tecnológica; y, si es verdad que nos hallamos ya en una Sociedad tecnológica, el tratamiento de la cuestión ética básica - bajo qué criterios orientar nuestras acciones y por qué- tiene ineludiblemente que habérselas con el fenómeno tecnológico tomado globalmente. No solamente por razones externas, sino por razones enraizadas en la misma entraña de la situación histórica, o, en términos más precisos, por la lógica interna de esa situación.

De entrada, es un lugar común referirse al fenómeno tecnológico como sistema tecnológico, es decir, como un todo internamente organizado con leyes autorreguladoras de funcionamiento específico, y en los que las partes se definen preferentemente por sus relaciones entre ellas y por su relación al todo. Ahora bien, esto significa que el mentado sistema tecnológico, como tal, ha de poseer una autonomía de funcionamiento que hace que pueda ser identificado como sistema ${ }^{2}$. Esta autonomía está reconocida hoy día como extremadamente intensa, tanto es así que incluso se ha acuñado en la literatura sobre Sociología y Filosofía de la Tecnología el término "imperativo tecnológico" para describir la fuerza intrínseca del sistema tecnológico. Dicho imperativo, que personalmente considero demasiado fuerte aunque puede aceptarse que señala con cierta justeza el poder social actual de la Tecnología, se formula así: "todo aquello que técnicamente se pueda hacer, se hará finalmente» ${ }^{3}$. 
Con todo, es cierto que el sistema tecnológico no es el sistema social global, pues se inscribe en éste como un subsistema ${ }^{4}$, pero su pujanza es sin duda impresionante; por eso, para responder, en la medida que sea posible, a la cuestión ética que todo el mundo plantea ante el avance tecnológico, es necesario tratar de introducirse dentro del sistema y ver cómo funciona. Pues es éste un requisito imprescindible si estamos tratando de plantear cómo introducir vectores éticos efectivos en la marcha del sistema tecnológico, e, indirectamente, en el sistema social global.

Para ello, resulta básico preguntar por el tipo de racionalidad interna que lleva consigo el sistema tecnológico. Es a este punto al que nos referíamos más arriba al apuntar el segundo rasgo de una Sociedad tecnológica. La descripción detallada de los caracteres de una racionalidad técnica la hemos desarrollado ya en varias de nuestras publicaciones al respecto ${ }^{5}$, por lo que aquí nos detendremos tan sólo en algunos de esos caracteres que incidan especialmente en la cuestión ética posterior.

En primer lugar, la racionalidad tecnológica es un tipo de racionalidad cuyo criterio epistemológico constituyente es la eficacia operativa, esto es, la actuación y manipulación concreta sobre la realidad, determinadas ambas unidireccionalmente por el fin técnico. $\mathrm{O}$, expresado en otros términos, la racionalidad tecnológica no busca prima facie la verdad del objeto en el sentido clásico (verdad teórica), sino un conocimiento concretamente encaminado a la modificación de lo real en una dirección específica y determinada por el fin técnico. Esta forma de racionalidad busca, por tanto, lo que se podría denominar una verdad pragmática, por diferenciación a la verdad teórica. Así, el criterio conductor del funcionamiento de una racionalidad tecnológica no intenta responder primigeniamente a la pregunta "¿qué es?", sino a la cuestión "ipara qué sirve el objeto?» La respuesta específica a este tipo de demandas es la que conforma el ejercicio de la racionalidad tecnológica.

Ahora bien, es necesario precisar un matiz importante a fin de evitar posibles malentendidos posteriores. El hecho de que el objetivo primordial de la racionalidad técnica sea la consecución de una verdad pragmática no significa que desaparezca de su ámbito la verdad teórica, pues ésta, lógicamente, también ocupa un lugar en su proceso cognoscitivo. Sobre todo si, además, tenemos presente que la racionalidad tecnológica moderna está especialmente ligada a la racionalidad científica, y ésta no duda en perseguir un tipo de verdad teórica propia y específica - aunque sea parcial, delimitada físicomatemáticamente, 
etc.- Más bien, lo que deseamos destacar fundamentalmente es que se produce una cierta inversión en los intereses cognoscitivos de la razón. Si antes la verdad teórica primaba como interés epistémico esencial y la verdad pragmática, que llevaba consigo la aplicación concreta del saber, quedaba subordinada a ella, ahora, en la racionalidad tecnológica, sucede al revés: la verdad pragmática, objetivo de la eficacia operativa, es la que ocupa el primer rango epistémico; y la verdad teórica quedará subordinada a ella, o sea, tendrá lugar en el proceso cognoscente en la medida en que sirva a los fines pragmáticos propuestos. Por eso, es cierto que la verdad teórica no desaparece de los procesos de la racionalidad tecnológica - sería absurdo pretenderlo-, pero su papel cambia ostensiblemente respecto del modelo tradicional moderno de racionalidad. Hasta cierto punto, se podría afirmar que la racionalidad tecnológica busca primordialmente que, introducidos determinados "inputs", se obtengan determinados "outputs", siendo subordinado el cómo y el porqué de esa obtención. Es decir, el primer nivel de importancia está marcado por la eficacia operativa del constructo obtenido, y no tanto por el saber teórico acerca del mismo, el cual, a pesar de ser necesario para el proceso cognoscitivo, no ocupa el puesto más importante como antaño.

En suma, la relación que encontramos en el modelo clásico entre verdad teórica y verdad pragmática cambia en la racionalidad tecnológica, y se podría afirmar que, como consecuencia final del criterio constituyente de eficacia operativa, la verdad teórica adquiere sólo un valor de instrumento para la verdad pragmática. En la racionalidad tecnológica, la theoria en sentido clásico queda en dependencia funcional de la praxis technica. Todo esto es causa de consecuencias muy importantes a nivel de la Epistemología de la Técnica, las cuales, obviamente, no podemos desarrollar en este momento ${ }^{6}$.

Un aspecto concreto de nuestro actual sistema tecnológico, que viene a ilustrar todo lo anterior, es justamente que la eficacia operativa mencionada se mide muy a menudo por la rentabilidad técnica y económica de los productos tecnológicos, es decir, no se exige sólo que la eficacia sea estrictamente tecnológica, sino que su proceso productivo además traiga consigo la máxima rentabilidad económica posterior, de tal modo que cualquier desarrollo o investigación teórica dentro del mismo se justificaría fundamentalmente por motivos de rentabilidad, la cual traduce, en el nivel socioeconómico, la idea general de eficacia operativa. Por ello, no sería arriesgado afirmar que el funcionamiento del subsistema económico en la actualidad es un claro ejemplo de racionalidad tecnológica, siendo quizás el subsistema social más semejante estructuralmente al sistema tecnológico. 
Un segundo rasgo de la racionalidad tecnológica, especialmente relevante, se deriva, en perfecta continuidad, de su criterio constituyente arriba esbozado. Se trata de que este tipo de racionalidad tiende naturalmente a una expansión indefinida. En efecto, pues el criterio de eficacia operativa implica subsumir el máximo posible de la realidad bajo dicha forma de racionalización. Si la eficacia operativa quiere ser verdaderamente eficaz, entonces tenderá continuamente a abarcar la mayor amplitud posible del mundo en donde se ubica históricamente. Sería una contradicción que la racionalidad tecnológica se autolimitara internamente en su ejercicio, al menos cuantitativamente, en lo referente a su expansión posible en la realidad. De ahí, por ejemplo, que cada vez tenga que haber más técnica, y cada vez el factor tecnológico, de modo general, adquiera una más grande relevancia social, histórica y política. Obsérvese, entonces, que el crecimiento sostenido de la Técnica, que se observa en nuestro mundo de hoy y es considerado desde falsas posiciones "irenistas" como una cierta amenaza, se debe no sólo a necesidades sociales, económicas, educativas, etc., sino a un carácter perteneciente a la misma estructura interna del fenómeno y del tipo de racionalidad implícito en él; es decir, al lado de importantes factores, por así decir, externos a la propia razón técnica y derivados de la situación histórica presente, que impulsan el desarrollo tecnológico, se ha de señalar la presencia de factores internos, derivados de la misma estructura de la racionalidad tecnológica que hacen ineludible su protagonismo cada vez más acentuado. De la misma manera que, en su momento de instauración histórica, la Ciencia no podía por menos que crecer, hoy, paralelamente, tendríamos que constatar que la Tecnología no puede por menos que aumentar permanentemente.

Los dos rasgos precedentes conducen derechamente a un tercero que cierra esta breve selección de caracteres de la racionalidad tecnológica, que, como mínimo, es necesario tener en cuenta para plantear más adelante la cuestión ética en sus justos términos. Esta tercera característica es que la racionalidad tecnológica, al aumentar su dominio epistemológico de modo natural y para reforzar su eficacia operativa, tenderá a organizarse sistémicamente. Ciertamente, porque la eficacia epistemológica se hará cada vez mayor mediante una interconexión de sus resultados, superando así la efectividad posible de tales resultados tomados cada uno aisladamente. Por el contrario, la tendencia natural habrá de ser el establecimiento de relaciones internas de feed-back entre los elementos propios y los resultados obtenidos en el ejercicio de la racionalidad tecnológica. Por eso, entre otras razones por supuesto, se han acuñado términos tales como "sistema tecnológico", "sistema 
de las acciones técnicas», etc., para describir la estructura del fenómeno tecnológico en la actualidad. Esta caracterización sistémica no es tan sólo una caracterización sociológica, o sea, un modelo explicativo extraído de la situación histórica efectiva, sino un rasgo que dimana de la estructura interna del propio fenómeno y del tipo de racionalidad que trae consigo.

Finalmente, y tomando prestada una antigua distinción filosófica - con el fin de hacer comprender mejor todo lo anterior-, se ha de rematar esta breve descripción de la racionalidad tecnológica destacando que los tres rasgos seleccionados no han de ser entendidos como un simple modus operandi; o sea, solamente como una forma epistémica de proceder cuyo origen radicara primordialmente en las condiciones históricas determinadas por el momento presente, sino que es preciso considerarlos como un modus essendi de la racionalidad tecnológica. Lo que significa que una racionalidad tecnológica, tal y como se halla presente en nuestros días, tenderá naturalmente a ser así, porque obviamente debe obedecer a su criterio constituyente y a su estructura interna. Ambos factores, es decir, factores externos e internos deben ser tenidos muy en cuenta. Normalmente, se acostumbra a cargar las tintas en los factores externos - históricos, sociales, etc.- del mundo de hoy, relegando los factores internos, los cuales han sido especialmente el objeto de nuestro análisis. Esta postergación origina una insuficiencia radical en la comprensión del sistema tecnológico actual, y, como consecuencia, una fragilidad de entrada en el planteamiento correcto de la cuestión ética.

\section{Metodología ética y sistema tecnológico}

$\mathrm{Si}$, de lo hasta aquí expuesto, se ha de destacar alguna consecuencia global respecto del fenómeno tecnológico, ésta sin duda puede ser que el sistema tecnológico ha de poseer una fortaleza intrínseca realmente grande para avanzar en su funcionamiento. Esta pujanza es un dato de partida, explícito o implícito, en la mayoría de los estudios de Filosofía y Sociología de la Técnica. La Tecnología, impulsada tanto por causas externas como por causas internas, se considera así como uno de los primeros elementos estructurantes de la realidad social, ya sea a nivel cuantitativo como cualitativo. De ahí, en buena medida, los temores frente a la influencia radical de la Tecnología en la vida humana: la así llamada muchas veces "invasión tecnológica", que llevaría al hombre a una situación vital de reduccionismo existencial acentuado ? 
Esta conciencia de la potencia del sistema tecnológico se acompaña, frecuentemente, de la idea de que el sistema posee un grado bastante elevado de autonomía en su funcionamiento, y que las posibilidades de reencauzar la dirección de éste son, en la práctica, muy reducidas. Ciertamente la idea de la autonomía propia del sistema tecnológico dentro del cuerpo social en general, posee un fundamento indiscutible, como se desprende de la descripción que hemos llevado a cabo en el parágrafo anterior y del hecho de que el desarrollo social y económico requiere hoy ineludiblemente el acceso general a la Tecnología. Ahora bien, aún reconociendo esa autonomía en grado relevante, no se ha de olvidar que el sistema tecnológico no es por sí solo el sistema social, sino un subsistema dentro de éste, y que, por tanto, está necesariamente abierto a relaciones e influencias de los otros subsistemas que también forman parte del sistema social. Por ejemplo, es un hecho que el subsistema político - los gobiernos- ejerce un influjo no despreciable sobre el funcionamiento de los demás subsistemas, y, entre ellos, el tecnológico ${ }^{8}$. Así, en una primera aproximación, si bien es cierto que la autonomía y la autopotencia del sistema tecnológico son un dato insoslayable, cuya ignorancia arruinaría cualquier propuesta ética eficaz, también se muestra la posibilidad, al menos téoricamente, de influir en él, dada su condición sistémica propia y su ubicación también sistémica dentro del conjunto del sistema social general. Esto significa que no se ha de caer en una actitud pesimista frente a las posibilidades éticas, sino más bien analizar cuidadosamente el asunto para encontrar una vía de penetración en el problema.

Porque, efectivamente, respecto a la necesidad actual de que el funcionamiento del sistema tecnológico se adecúe éticamente a los problemas humanos de toda índole, nadie duda seriamente. Establecer instancias éticas, valores, etc., para dirigir el desarrollo tecnológico e, incluso, la innovación técnica, es un tema ciertamente debatido y consensuado en la actualidad.

Ahora bien, el problema reside no en el "qué» - pues hay unanimidad en ello- sino en el "cómo" hacerlo, para que el asunto llegue a buen término. Sobre esto existen propuestas de lo más variado, que no podemos describir detalladamente aquí ${ }^{9}$, pero de las cuales sí es procedente señalar un defecto fundamental, a saber, que enfrentan la instancia ética -0 , si se prefiere, el subsistema ético- al sistema tecnológico, concibiéndose la realidad del problema como una situación de combate entre lo ético y lo tecnológico. Esta actitud se refleja incluso en el lenguaje más comúnmente utilizado para expresar ese deseo ético: se habla, por ejemplo, de poner "límites" a la Tecnología, de 
"frenar" la invasión tecnológica, etc. Es una posición que contempla el problema como si fuera la batalla entre dos ejércitos bien diferenciados, y en una relación casi de David a Goliath, siendo éste último, por supuesto, el sistema tecnológico.

Esta forma de plantear la cuestión ética está abocada al fracaso más rotundo. Justamente por lo señalado al comienzo, o sea, la fortaleza y la relativa autonomía del sistema tecnológico, la cual no va a admitir de buen grado ninguna instancia, sobre todo externa, ya sea instancia ética o no, que menoscabe su funcionamiento eficaz. Y la razón de ello ya la conocemos: porque el sistema no puede contradecirse a sí mismo, y si el criterio constituyente de su lógica interna es la eficacia operativa, entonces jamás va a aceptar la intromisión de un elemento ajeno a él que aminore el cumplimiento de sus fines. Sencillamente, no puede; pues sería, por así decirlo, ir en contra de su propia naturaleza. Además, otras instancias sociales interesadas -grupos de presión de todo tipo, ponemos por caso-, reforzarían ostensiblemente esta posición.

Lo interesante en este punto del análisis es percatarse de que, no sólo por factores sociales en cierta manera externos al sistema tecnológico, sino que también por la propia estructura interna del sistema, esa Metodología de enfrentamiento, que pretende una subordinación ética de su funcionamiento por motivaciones externas, choca frontalmente con la potencia reconocida del sistema tecnológico, siendo el resultado de este choque una derrota y un aplastamiento de las instancias éticas; lo cual, además, supondrá indirectamente un reforzamiento de la autonomía del sistema tecnológico. El criterio de la eficacia operativa, al cual debe obedecer el sistema, recuérdese, como a un modus essendi, impedirá, en la práctica, cualquier interferencia que se desee introducir en la lógica interna del sistema.

Entonces, ¿qué? ¿Se dirá que no existe posibilidad viable para introducir, al menos, algunos vectores éticos convenientes y deseables en el sistema tecnológico, dejándolo a su aire y teniendo que soportar sus consecuencias? ¿O, a lo sumo, tendremos que contentarnos con dejar el asunto solamente en manos de políticos más o menos bienintencionados que ocupen lugares decisorios? Parecería que nos encontrásemos en una callejón sin salida. Pues, efectivamente, de una parte, nadie estará dispuesto a renunciar ni al avance tecnológico ni a sus posibles aplicaciones sociales, ya que el buen asentamiento del hombre en el mundo depende de ellos ${ }^{10} ; \mathrm{y}$, de otra parte, el sistema tecnológico posee una autonomía y una potencia que dificultan extremadamente la eficiencia posible de una acción ética, y que podrían 


\section{Ramón Queraltó}

conducirnos a situaciones de cierto peligro moral y ético-social (piénsese, por ejemplo, en el reciente caso de la clonación de seres humanos).

Afortunadamente creemos que no es así. Puede existir una vía posible de solución del problema, al menos según los términos en que ha sido planteado. Si es verdad que el sistema tecnológico, globalmente considerado, posee esa pujanza funcional y esa expansión indefinida señalada arriba, entonces la única manera plausible de introducir instancias externas, vectores éticos, en el sistema será a la manera tecnológica, o sea, siguiendo las pautas directrices propias, a las que debe obedecer forzosamente; porque, de ese modo, el sistema no podrá rechazarlas sino acogerlas dentro de sí, precisamente -y otra veza causa de su lógica interna basada en el criterio constituyente de eficacia operativa.

Ahora bien, ¿qué significa esta Metodología para la dimensión ética? Pues mostrar que el vector ético es eficaz para el propio funcionamiento del sistema tecnológico, que la Etica - y sus aplicaciones concretaspuede hacer funcionar más eficazmente el sistema, que aceptar el vector ético es conveniente para él en aras de una mayor eficacia propia. En definitiva, que la Etica es útil, en el sentido tecnológico, es decir, que puede contribuir a mejorar los fines estrictamente técnicos del sistema. Así, de acuerdo con la propia lógica del sistema, la instancia ética podrá tener éxito. Se podría denominar a esta propuesta «la tesis del caballo de Troya al revés», ya que se introduce a un "presunto enemigo", que después no sólo no destruye sino que ayuda a mejorar el estado inicial. Se trata, por tanto, de destacar el aspecto pragmático de los vectores éticos deseados, haciendo ver que lo ético como tal es también eficaz.

Muy pocas veces se observa un comportamiento de este género en nuestro mundo actual. Lo habitual es presentar a la instancia ética enfrentada a la "maldad posible" del sistema tecnológico, pero en rara ocasión se descubre la vertiente útil de la dimensión ética. En buena medida, se trataría de hacer visible aquella vieja idea de que el comportamiento ético es bueno para el hombre porque le causa bienestar existencial, no sólo espiritual y psicológico sino incluso simplemente físico; se trataría de recuperar esa vertiente de utilidad material que la Etica ha poseído desde siempre, y que los planteamientos intelectualistas de la cuestión moral - la influencia de la fundamentación kantiana, por ejemplo, no es desdeñable - han dejado progresivamente de lado.

Ahora bien, caben sin duda objeciones interesantes a este principio metodológico, que deben ser examinadas con atención. 
En primer lugar, se podrá argüir que ésta es una idea teórica cuya posibilidad práctica es muy dudosa, no dejando de ser otro «sueño dogmático". Sin embargo, esta objeción no se sostiene, puesto que ya hay ejemplos de introducción de instancias éticas con resultados concretos, en aras a la eficacia global de una determinada función técnica. Y citaremos algo aparentemente muy modesto y casi banal, pero que sin la menor duda ha supuesto una mejora importante en las condiciones laborales: la introducción de la climatización - aire acondicionadoen la mayoría de las oficinas de gestión y en los bancos ubicados en regiones con elevadas temperaturas. ¿Acaso se hizo esto por razones de la fuerza moral que acompañaba la solicitud de tal mejora? Nada de eso. Se llevó a cabo porque la eficacia laboral aumentaba, y de hecho así se comprobó posteriormente de manera lineal y casi determinista. Fueron, claramente, razones economicistas las que impulsaron al subsistema económico-bancario a modificar esas condiciones laborales, pero trajeron como consecuencia un mayor bienestar en el trabajo. Aunque éste sea un ejemplo de alcance reducido - se podría ampliar el elenco sin duda ${ }^{11}$-, muestra cómo debería ser la Metodología ética eficaz, en el momento presente, para tratar de superar los posibles peligros de un funcionamiento autónomo y potente del sistema tecnológico. Dicha Metodología es la que se comienza a emplear en organismos tales como los Foros de Mediación, nacionales e internacionales, cuyos principios de actuación incorporan diversos vectores éticos para la resolución de conflictos interempresariales mostrando su eficacia real. En definitiva, se trata de recuperar el valor de eficacia que siempre acompañó a la teorización de la Etica e introducirlo, a la manera del "caballo de Troya", en el sistema tecnológico.

Otra objeción importante es, sin duda, que si se presenta la Etica en función de su "utilidad", se corre el peligro de una instrumentalización y manipulación de la propia Etica, pues sería reducir su relevancia social y antropológica a la de una justificación utilitarista. En efecto, es de justicia reconocer que ese peligro en la práctica existe, si bien pueden añadirse algunas puntualizaciones oportunas. En primer lugar, en la Metodología indicada en ningún caso se ha de entender que la dimensión ética se reduzca a su utilidad pragmática; en ningún momento se ha de perder de vista que la fundamentalidad de la Etica para la vida del hombre no puede justificarse sólo desde un punto de vista utilitarista. Es cierto que tampoco se debe menospreciar este nivel de lo ético, pero, en las razones de la obligación moral, debe sin duda ser destacada la justificación de los principios y valores éticos básicos por sí mismos, en cuanto pueden poseer una validez intrínseca enraizada 
en la dignidad radical del hombre entendido como ser personal. Por eso, la aceptabilidad de un determinado principio de actuación ética no puede reducirse a su utilidad pragmática dentro del sistema tecnológico. La Metodología apuntada significa primariamente una estrategia para introducirse en el sistema tecnológico con sus propias armas y aprovechando sus características internas, puesto que, si no fuera así, sería prácticamente imposible reencauzar la marcha del sistema tecnológico, a causa de su potente autonomía y su rechazo a recibir instancias ajenas en su seno. En este sentido, sería preciso delimitar con todo detalle los límites críticos de la actuación ética en el sistema, por debajo de los cuales no sería procedente ese uso estratégico del vector ético, debiéndose pasar entonces a otras instancias - por ejemplo, la acción política estricta-. Todavía se tendrá ocasión de ampliar algo más este punto en el próximo parágrafo.

Abundando en esta línea, se trataría asimismo de clarificar que la utilidad de la Etica se entiende aquí en el sentido de restituirle aquella dimensión de "Etica para la felicidad", que se hallaba ya en los comienzos de la Filosofía -recuérdese sin más a Aristóteles-, aspecto de lo ético que, sin duda, se ha reducido sobremanera en la actitud existencial del hombre actual. Se denuncia hoy con insistencia que para la Sociedad contemporánea - y también para el propio hombre-, la Etica se ha convertido en una instancia molesta, incluso fastidiosa, que dificulta la libre expresión de la vida y de la creatividad humanas. Esto, obviamente, no tiene que ser así necesariamente. Antes bien, existe toda una tradición Etica de doctrinas eudemónicas que destacan la felicidad como fin de la vida moral, y, por tanto, de las leyes y mandatos morales. Por ello, destacar la vertiente de utilidad de la Etica no tiene por qué concebirse en sentido reductivo utilitarista, sino que cabe dentro de una Etica para la felicidad, la cual posee muchas dimensiones diferentes - física, psicológica, etc.-, y a las cuales, precisamente, tanto ha contribuido y ha de contribuir todavía la misma Tecnología, en virtud de su finalidad propia. Así, cabría incluso la posibilidad de proponer una Etica material, no ya formal, basada en la utilidad, sin que se tuviera que caer en una manipulación instrumentalista de los vectores éticos que se propusieran.

En segundo lugar, hay que decir que el hecho mismo de que el sistema tecnológico sea, a fin de cuentas, un subsistema del sistema social global, y también pueda hablarse de la instancia ética como configuración sistémica - subsistema ético-, aminora en cierto sentido el riesgo, por otra parte real, de la instrumentalización reduccionista de la Etica. Pues, en efecto, en perspectiva sistémica, el ideal del 
sistema social será alcanzar la optimización de la totalidad, y esto no puede conseguirse si en el proceso se elimina un subsistema propio e irreductible del sistema en su conjunto, o si se le rebaja a un nivel inferior a su intervalo crítico ${ }^{12}$. Si esto ocurriera, las señales de alarma aparecerían de inmediato, como, por ejemplo, parece haber ocurrido en la actualidad con la prohibición por los gobiernos occidentales de ciertas experimentaciones biogenéticas. Todo ello quiere significar que, a pesar del bajo nivel social de la dimensión ética en la actualidad respecto del que sería más deseable, los indudables peligros de un reduccionismo utilitarista de los vectores éticos no son imposibles de evitar y de limitar sus posibles efectos nocivos. Con esto no se quiere afirmar que el riesgo desaparezca o sea mínimo, pues eso, en cuestiones humanas, sería ilusorio pretenderlo; más bien queremos indicar que dicho riesgo, que siempre existirá, es un riesgo razonable y asumible, ya que, por una parte, ha sido puesto al descubierto, y por otra, se han indicado las posibles razones para una reacción oportuna del sistema social.

\section{Materiales de los vectores éticos}

Indicado con la brevedad necesaria al caso el modo de introducción de los vectores éticos en el sistema tecnológico, debemos terminar apuntando al menos cuáles podrían ser los hilos conductores generales de dichos vectores a transmitir al sistema. Es obvio que este tema, y sobre todo su fundamentación, requeriría mucho mayor detenimiento, así que nos limitaremos a establecer, y justificar, algunas ideas que han de funcionar como conditio sine qua non en la cuestión señalada.

Nuestra estrategia metódica se concretará, de acuerdo con lo dicho más arriba, en buscar vectores éticos cuya estructura y posible funcionamiento social presenten inicialmente una cierta homomorfía con la estructura y funcionamiento propios del sistema tecnológico. Así, su justificación vendrá dada por razones endógenas al mismo sistema tecnológico, evitando cualquier tipo de enfrentamiento directo con él, ya que tal método se ha mostrado antes que sería inviable. Por consiguiente, no se trata de argüir a partir de la bondad y justicia intrínsecas de los valores éticos, que sería una justificación doctrinal -la cual, no obstante, hallará su lugar adecuado más adelante-, sino desde la eficacia operativa posible de tales valores. Y para ello, parecería plausible buscar esa homomorfía mencionada. 
En primer lugar, un vector ético esencial se deriva, directamente de nuevo, de la condición sistémica del fenómeno tecnológico y también de la condición sistémica de la organización social: la optimización del sistema tecnológico; puesto que es a fin de cuentas un subsistema del sistema social, no podrá alcanzarse convenientemente sin la presencia del principio de solidaridad. $\mathrm{O}$, dicho de otro modo, entre la solidaridad como dimensión moral y la interrelación sistémica se puede establecer una cierta semejanza estructural que permitiría la estrategia metodológica arriba reseñada. En este sentido, habría que mostrar que la conectividad sistémica, y, por tanto, la fuerza del sistema, se reforzaría con la solidaridad soeial, la cual al propio tiempo podría introducir elementos de mejora existencial para los individuos humanos afectados en su caso, sin excesiva resistencia por parte del sistema, ya que a éste también le interesaría aumentar su capacidad de eficacia.

Este vector de solidaridad se impone, además, por la globalización de nuestro mundo a final del siglo XX. La complejidad, como categoría ontológica, es una de las características esenciales de la Sociedad tecnológica ${ }^{13}$, lo cual hace que las distorsiones y desencajes, incluso pequeños, del sistema social puedan tener efectos devastadores para el propio sistema - lo que obviamente no le conviene, pues se opondría flagrantemente al criterio de eficacia operativa-; por lo que, con tanta mayor razón, es preciso reivindicar el vector solidaridad como principio vertebrador del conjunto. Esta situación, que hasta hace poco no existía en el planeta, es típica de una Sociedad mundializada como la actual, en la que la complejidad ha adquirido el rango de rasgo estructural. Es preciso, pues, indagar, para mostrarla después, en la dimensión técnico-pragmática de la solidaridad como coadyuvante a los fines globales y sectoriales de los diferentes sistemas tecnológicos concretos de la Sociedad actual.

Como consecuencia de la solidaridad, se imponen otros dos vectores que son imprescindibles para la realización efectiva de la misma, es decir, dos vectores que funcionarían casi como condiciones de posibilidad del vector solidaridad. Son, de un lado, la igualdad de derechos, y, de otra parte, la justicia social. Los tres conforman lo que se podría denominar el triedro ético fundamental. En efecto, la solidaridad, en cuanto idea ética teórica requiere, para no quedarse en una simple declaración de buenas intenciones, la igualdad jurídica de los individuos y de los grupos sociales diversos que actúen como unidades sociales en cada circunstancia. Obsérvese que no se trata de un "igualitarismo social", que ya se ha demostrado inviable en la práctica (recuérdese el fracaso histórico reciente de los colectivismos). Se trata, más bien, 
del viejo vector de la igualdad de derechos que lleva consigo una igualdad de oportunidades, la cual, a su vez, es fácil ver que supone una condición básica para la consecución en la práctica de la solidaridad. De no existir efectivamente tal igualdad, la experiencia histórica ha mostrado que, en las sociedades democráticas occidentales, se llega indefectiblemente a una inestabilidad social acusada, que afectaría notablemente a la optimización del sistema tecnológico, pues la distorsión del sistema social en su conjunto impediría el logro de, al menos, fines técnicos sectoriales, obteniéndose un resultado inferior sin duda a la optimización asequible. Por supuesto, no queremos decir que el sistema tecnológico se autodestruyese - lo que es casi imposible - o no funcionase de alguna forma, sino que tal funcionamiento estaría lejos del nivel de optimización posible, y es ese, justamente, el punto de referencia desde el que se ha de entender este análisis.

El vector restante, justicia social, se remite especialmente - aunque no sólo- a la distribución de los beneficios del sistema tecnológico, y parece claro que sin su presencia se desembocaría fácilmente de nuevo en una situación de inestabilidad, cuyas consecuencias problematizarían ese punto capital de referencia que es su optimización. En suma, la situación presente con su carácter de Sociedad mundializada hace inviable, precisamente para su optimización, una independencia de facto del sistema tecnológico, pues justamente el altísimo grado de conectividad social exige su integración sistémica global con los demás subsistemas, entre los cuales se halla lógicamente el subsistema ético.

La labor fundamental consiste ahora en mostrar esa eficacia intrínseca posible de los vectores éticos en relación al sistema tecnológico y respecto a situaciones concretas. El análisis teórico de la cuestión parece mostrar la viabilidad posible de la tesis, pero indudablemente se requiere analizar ejemplos de situaciones sociales determinadas en las que se muestre la eficacia operativa de los vectores éticos. Algunos de ellos han sido aludidos a lo largo de esta contribución, en hipotético apoyo de esta propuesta.

También hay que advertir que la indicación del triedro ético anterior no pretende ser exhaustiva; se trata solamente de descender con un poco más de detalle desde el nivel puramente teorético para proponer qué principios éticos podrían considerarse inicialmente. No es éste el lugar para realizar dicho camino «descendente», sino tan sólo de mostrar su posible viabilidad teórica razonando el porqué básico de los tres vectores propuestos, y, muy especialmente, el vector inicial, o sea, el vector solidaridad. 
Hemos de reiterar que la propuesta del triedro ético señalado sale del análisis realizado a causa de la Metodología propuesta antes, o sea, por motivos de eficacia operativa; no se trata, pues, de una justificación doctrinal, la cual, por supuesto es posible y, además, necesaria para evitar el peligro de instrumentalización reductiva de la instancia ética. Pero es imprescindible diferenciar netamente ambos polos del problema: la justificación según la utilidad deriva de un análisis interno de la estructura del sistema tecnológico, y buscando su optimización con sus propias armas fundadas en la eficacia operativa; es desde ahí desde donde se ha de proponer la introducción de los vectores éticos, y también desde ahí desde donde se han de obtener los contenidos oportunos de dichos vectores (solidaridad, etc.). Así, la justificación ética será una justificación operativa que el sistema, al menos en principio y con muy alta plausibilidad, no podrá rechazar sin caer en contradicción.

Ahora bien, de otra parte está la justificación doctrinal de la instancia ética y de sus vectores, es decir, la que provendría de un análisis externo al propio sistema como tal, la cual es necesaria tenerla presente, no para enfrentarla al sistema tecnológico, pues eso llevaría al fracaso ético-social y al fortalecimiento no deseable del "imperativo tecnológico", sino para evitar el reduccionismo instrumentalista de lo ético y tener siempre a la vista la especificidad de lo humano y sus exigencias intrínsecas respecto a lo técnico y su organización actual. Pero, insistimos, la Metodología eficaz aconseja utilizar esta justificación doctrinal, clásica, para este cometido vigilante que alertara ante la gestación de cualquier situación inaceptable antropológicamente, y no enfrentarla directamente al sistema tecnológico, pues, por las razones apuntadas más arriba, no sólo se fracasaría sino que se conseguiría de hecho un resultado contrario al que se estaría pretendiendo.

Finalmente, hay que indicar al menos que esta justificación doctrinal también debe adaptarse a la situación histórica presente, caracterizada por la mundialización social y la complejidad estructural. A tal respecto, la construcción de un nuevo humanismo que tenga en cuenta las condiciones históricas específicas en las que se halla el hombre de principios del tercer milenio se impone necesariamente. Tal humanismo implicaría una triple tarea: reflexión filosófica sobre el ser del hombre en su ubicación histórica presente, propuesta de valores sociales derivados de tal reflexión previa, y transmisión social del nuevo humanismo a través de los sistemas educativos. De este modo, y tan sólo a título de ejemplo, indicaríamos que, respecto a la primera tarea, un capítulo heredado y esencial es sin duda aquella doctrina que se encuentra 
tradicionalmente anclada en el concepto de persona aplicado al ser humano. Indudablemente, tal concepción posee muchos elementos intrínsecamente valiosos y, casi se diría, de validez intemporal. Ahora bien, qué duda cabe igualmente que tales valores de la persona adquieren su significación efectiva en coordenadas históricas determinadas. Dado que nos hallamos en un cambio de época histórica y a las puertas de una nueva estructura social mundial condicionada fuertemente por la Tecnología, y, más concretamente, por las Tecnologías de la información, se hace necesaria una revisión teórica del concepto de persona, no tanto para modificarlo en su sustancialidad, sino más bien para todo lo contrario, es decir, hacerlo efectivo en esa Sociedad mundializada, que es un evento nuevo en la Historia de la Humanidad. Esta tarea está aún por realizar ${ }^{14}$, y es además tarea de urgencia, pues, de no llegar a tiempo, se estaría colaborando a un desfase todavía mayor entre lo ético y lo tecnológico, entre lo ético y lo social, entre lo ético y lo político, en la Historia que nos ha tocado vivir; circunstancia ésta de la que hemos partido como dato histórico y experimental señalado unánimemente en la actualidad. Ahora bien, tales cometidos no pueden corresponder ya a la medida de la presente contribución, cuyo objetivo fundamental, no se olvide al final, ha sido el de esbozar una posible estrategia práctica para hacer más efectiva la presencia de los factores éticos en la Sociedad tecnológica.

\section{Notas}

1 Véase al respecto, CASTElls, M., La Era de la Información: Economía, Sociedad y Cultura. Vol. I: La Sociedad Red. Vol. II: El Poder de la Identidad; Vol. III: Fin del Milenio, Alianza Ed., Madrid, 1997-98.

2 La bibliografía sobre teoría de sistemas es inmensa. Al respecto citaremos sólo las siguientes obras: BERTALANFFy, L. VoN, Teoría general de los sistemas, F.C.E., México, 1976; Bertalanffy, L. Von, Perspectivas en la teoria general de sistemas, Madrid, Alianza Ed., 1979; BUCKLEY, W. (ed), Modern System Research for the Behavioral Scientist, Aldine, Chicago, $5^{\text {a }}$ ed., 1976; LASzLo, E., Introduction to Systems Philosophy (con prólogo de L. von Bertalanffy), Gordon and Breach, N. York, $2^{\mathrm{a}}$ ed., 1984; ARACIL, J., Máquinas, sistemas, modelos, Alianza Ed., Madrid, 1986.

3 Uno de los autores que más han incidido sobre este tema, ciertamente con un pesimismo exagerado, es J. Ellul, cfr. Ellul, J., La technique ou l'enjeu du siècle, Oeconomica, París, 1990 (reimp. de 1954); y Ellul, J., Le système technicien, Calman-Levy, París, 1977.

4 Véase una brillante descripción detallada de este punto en AGAzZı, E., El bien, el mal, y la Ciencia. Las dimensiones éticas de la empresa cientifico-tecnológica, edición a cargo de Ramón Queraltó, Tecnos, Madrid, 1996, cap. VI, pp. 129-154. 
5 Cfr. Queraltó, R., Mundo, Tecnología y razón en el fin de la Modernidad. ¿Hacia el hombre "more technico»?, P.P.U., Barcelona, 1993; QUERALTÓ, R., "La razionalità tecnica come mediazione epistemologica tra l'uomo contemporaneo ed il reale", en AGAzZI, E. (ed), Interpretazioni attuali dell'uomo, Atti del Convegno dell'Académie Internationale de Philosophie des Sciences, Guida Editori, Nápoles, 1995, pp. 239. 248; Queraltó, R., "Razón técnica e idea de verdad: ¿una oposición inevitable?", en Dou, A. (ed), Evaluación social de la Ciencia y de la Técnica, Publicaciones de la Universidad Pontificia Comillas, Madrid, 1996, pp. 19-46; y QUERALTO, R., Ra. zionalità tecnica e mondo futuro: un'eredità per il terzo millennio, Ed. Piemme, Casale Monferrato, 1998.

6 A este respecto, el lector interesado puede consultar los siguientes trabajos: Queraltó, R., "Does Technology 'constructi' Scientific Reality?", en Mitcham, C. (ed), Philosophy of Technology in Spanish Speaking Countries, Kluwer, Dordrecht-Boston, 1993, pp. 167-172; QUERALTo, R., "Hypothèse, objectivité et rationalité technique", Philosophia Scientiae (Revue publiée par les Archives-Centre d'Etudes H. Poincaré, Nancy), vol. I, Cahier Spécial 1, (1996), Entretiens de la session 1994 de l'Académie Internationale de Philosophie des Sciences, pp. 187-196; y QUERALTÓ, R., "Scientific Realism, Objectivity and Technological Realism'”, en AGAZZI, E. y PAURI, M. (eds), The Reality of the Unobservable. Observability, Unobservability and their impact on the Issue of Scientific Realism. Kluwer, Dordrecht, 1999.

7 Quizás el "profeta pesimista" más conocido respecto a este asunto sea el ya mencionado J. Ellul, que insiste sobremanera en esta línea, desde sus primeras obras así como en la más reciente: Ellul, J., Le blouff technologique, Hachette, París, 1988.

8 Piénsese al respecto en la definición por parte de los organismos gubernativos de "lineas prioritarias de investigación", programas de I+D, subvenciones a determinadas líneas de actuación tecnológica 0 , incluso, la creación de mercados específicos para la salida de productos tecnológicos de fabricación propia. Sobre el papel de los organismos políticos en la Era tecnológica, véase CASTELLS, M., La Era de la Información: Economía, Sociedad y Cultura. Vol. I: La Sociedad Red, pp. 79 ss.

9 Por ejemplo, la intervención del gobierno, los pactos internacionales, la sumisión a un tribunal internacional, la obediencia debida a organismos internacionales representativos, etc.

10 Esta afirmación no se hace sólo desde una dimensión histórica, sino también desde una dimensión ontológica, puesto que consideramos que la Técnica es una mediación fundamental -en sentido ontológico- para el asentamiento del hombre en la realidad. Pueden consultarse a este respecto nuestros trabajos ya citados en la nota 5 .

11 Por ejemplo, es sintomático que la C.E.O.E. (Confederación Española de Organizaciones Empresariales) esté promoviendo en lo últimos años con asiduidad seminarios sobre temas como la Etica en la empresa, la calidad total como meta -que incluye no pocas connotaciones éticas en la actividad económica, etc.-. Y, ciertamente, lo que impulsa a este organismo social no es precisamente una instancia ética en primer término, sino el principio de eficacia operativa para alcanzar la máxima rentabilidad de la actividad económica.

12 Véase el detallado estudio que Agazzi realiza de este asunto en AGazzi, E., El bien, el mal, y la Ciencia, cap. XII, pp. 298-329.

13 La bibliografía acerca de la complejidad es muy numerosa, por lo que nos limitaremos a indicar un libro-fuente (source book) en el que pueden encontrarse estudios 


\section{Cómo introducir vectores éticos eficaces en el sistema...}

fundamentales sobre este tema: BOCCHI, G. y CERUTI, M. (eds), La sfida della complessità, Feltrinelli, Milán, $8^{\text {a }}$ ed., 1995.

14 Es, por ejemplo, uno de los cometidos emprendidos (entre otros) dentro del Grupo de Investigación (D.G.I.C.Y.T., PB96-1351) que dirijo en la Universidad de Sevilla.

\section{Bibliografía}

Agazzı, E., El bien, el mal y la Ciencia. Las dimensiones éticas de la empresa científico-tecnológica, edición a cargo de Ramón Queraltó, Tecnos, Madrid, 1996.

Agazzi, E., La techno-science et l'identité de l'homme contemporain, Éditions de l'Université Fribourg, Friburgo (Suiza), 1997.

Aracil, J., Máquinas, sistemas, modelos, Alianza Ed., Madrid, 1986.

Bertal.anffy, L. von, Teoría general de los sistemas, F.C.E., México, 1976.

Bertalanffy, L. von, Perspectivas en la teoría general de sistemas, Alianza Ed., Madrid, 1979.

Boccill, G. y Cerutı, M. (eds), La sfida della complessità, Feltrinelli, Milán, $8^{a}$ ed., 1995.

Buckl.ey, W. (ed.), Modern System Research for the Behavioral Scientist, Aldine, Chicago, $5^{\mathrm{a}}$ ed., 1976.

Bunge, M., Epistemology and Methodology III: Philosophy of Science and Technology, en Bunge, M., Treatise on Basic Philosophy, vol. 7, Reidel, Dordrecht, 1985.

CASTElls, M., La Era de la Información: Economía, Sociedad y Cultura. Vol. I: La Sociedad Red. Vol. II: El Poder de la Identidad; Vol. III: Fin del Milenio, Alianza Ed., Madrid, 1997-98.

Durbin, P. (ed), Technology and Contemporary Life, Reidel, Dordrecht, 1978.

Ellul, J., La technique ou l'enjeu du siècle, Oeconomica, París, 1990 (reimp. 1954).

Ellul, J., Le système technicien. Calman-Levy, París, 1977.

Ellul, J., Le blouff technologique, Hachette, París, 1988.

Feibleman, J. K., Technology and Reality, M. Nijhoff, La Haya, 1982.

Garcta Bacca, J. D., Elogio de la Técnica, Anthropos, Barcelona, 1987.

GibBons, M. y Gummetr, P. (eds), Science, Technology and Society Today, University of Chicago Press, Chicago, 1984.

Jonas, H., The Imperative of Responsibility: In Search of an Ethics for the Technological Age, Chicago University Press, Chicago, 1984.

Laudan, R. (ed.), The Nature of Technological Knowledge, Reidel, Dordrecht, 1984.

Laszio, E., Introduction to Systems Philosophy (con un prólogo de L. von Bertalanffy), Gordon and Breach, N. York, $2^{\mathrm{a}}$ ed., 1984.

Lenk, H., Zur sozialphilosophie der Technik, Suhrkamp, Francfort, 1982.

Lenk, H., Macht und Machbarkeit der Technik, Reclam, Stuttgart, 1994.

Mayz Vall.enili. , E., Ratio technica, Monte Avila, Caracas, 1983.

Mayz Vallienil.ia, E., Fundamentos de Meta-técnica, Gedisa, Barcelona, 1993.

Mitcinm, C. y Hunging, A. (eds), Philosophy and Technology II: Information Technology and Computers in Theory and Practice, Reidel, Boston, 1986.

Mumrori, L., Técnica y civilización, Alianza Ed., Madrid, 1971.

Queraitó, R., "La dimensión epistemológica del uso tecnológico en el proceso de conocimiento científico", Thémata, v. 9, (1992), pp. 289-297. 
Queralto, R., “Does Technology 'construct' Scientific Reality?", en Miтchaм, C. (ed), Philosophy of Technology in Spanish Speaking Countries, Kluwer, Dordrecht, 1993, pp. 167-172.

Queraltó, R., Mundo, Tecnología y razón en el fin de la Modernidad. ¿Hacia el hombre "more technico«?, P.P.U., Barcelona, 1993.

Queralto, R., "La razionalità tecnica come mediazione epistemologica tra l'uomo contemporaneo ed il reale", en Agazzi, E. (ed), Interpretazioni attuali dell'uomo, Atti del Convegno dell'Académie Internationale de Philosophie des Sciences, Guida Editori, Nápoles, 1995, pp. 239-248.

Queralto, R., "Razón técnica e idea de verdad: ¿una oposición inevitable?», en Dou, A. (ed), Evaluación social de la Ciencia y de la Técnica, Publicaciones Universidad Pontificia Comillas, Madrid, 1996, pp. 19-46.

Queralto, R., "Hypothèse, objectivité et rationalité technique", Philosophia Scientiae (Revue publiée par les Archives-Centre d'Etudes H. Poincaré, Nancy), vol. I, Cahier Spécial 1, (1996), Entretiens de la session 1994 de l'Académie Internationale de Philosophie des Sciences, pp. 187-196.

Queralto, R., "Since Indeterminacy: the New Picture of the Physical World at the End of Modernity", en Ginev, D. y CoHen, R. S. (eds), Issues and Images in the Philosophy of Science. Kluwer, Dordrecht, 1997, pp. 253-262.

Queraltó, R., Razionalità tecnica e mondo futuro. Un'eredità per il terzo millennio, Ed. Piemme, Casale Monferrato/Milán, 1998.

Queralto, R., "Scientific Realism, Objectivity and 'Technological Realism', en Agazzı, E. y PAURI, M. (eds), The Reality of the Unobservable. Observability, Unobservability and their impact on the Issue of Scientific Realism, Kluwer, Dordrecht, 1999.

SArtori, G., Homo videns. La Sociedad teledirigida, Taurus, Madrid, 1998.

Sanmartin, J., Tecnología y futuro humano, Anthropos, Barcelona, 1990.

VArios Autores, Identidad cultural, Ciencia y Tecnología, G. Cambeiro, Ed., Buenos Aires, 1987.

VariosAutores, "Tecnología, Ciencia, Naturaleza y Sociedad", Anthropos, n 94-95, (1989), (número preparado por el "Invescit").

Varios Autores, Sociología de la Ciencia y de la Tecnología, coord. por J. M. Iranzo, J. R. Blanco, T. González de la Fe, C. Torres y A. Cotillo, Consejo Superior de Investigaciones Científicas, Madrid, 1995.

Varios Autores, Ciencia, Tecnología y Sociedad, edición de M. I. González, J. A. López Cerezo y J. L. Luján, Ariel, Barcelona, 1997.

WinNer, L., La ballena y el reactor. Una búsqueda de los limites en la Era de la alta Tecnología, Gedisa, Barcelona, 1987. 